\title{
Coupling a polarizable water model to the hydrated ion-water interaction potential: A test on the $\mathrm{Cr}^{3+}$ hydration
}

\author{
José M. Martínez \\ Departamento de Química Física, Universidad de Sevilla, 41012-Sevilla, Spain \\ Jorge Hernández-Cobos and Humberto Saint-Martin \\ Centro de Ciencias Físicas, Universidad Autónoma de México, Apartado Postal 48-3, Cuernavaca 62251, \\ México \\ Rafael R. Pappalardo \\ Departamento de Química Física, Universidad de Sevilla, 41012-Sevilla, Spain \\ Ivan Ortega-Blake \\ Centro de Ciencias Físicas, Universidad Autónoma de México, Apartado Postal 48-3, Cuernavaca 62251, \\ México \\ Enrique Sánchez Marcos ${ }^{a)}$ \\ Departamento de Química Física, Universidad de Sevilla, 41012-Sevilla, Spain
}

(Received 28 June 1999; accepted 28 October 1999)

\begin{abstract}
A strategy to build interaction potentials for describing ionic hydration of highly charged monoatomic cations by computer simulations, including the polarizable character of the solvent, is proposed. The method is based on the hydrated ion concept that has been previously tested for the case of $\mathrm{Cr}^{3+}$ aqueous solutions [J. Phys. Chem. 100, 11748 (1996)]. In the present work, the interaction potential of $\left[\mathrm{Cr}\left(\mathrm{H}_{2} \mathrm{O}_{6}\right)\right]^{3+}$ with water has been adapted to a water model that accounts for the polarizable character of the solvent by means of a mobile charge harmonic oscillator representation (MCHO model) [J. Chem. Phys. 93, 6448 (1990)]. Monte Carlo simulations of the $\mathrm{Cr}^{3+}$ hexahydrate plus 512 water molecules have been performed to study the energetics and structure of the ionic solution. The results show a significant improvement in the estimate of the hydration enthalpy $\left[\Delta H_{\mathrm{hydr}}\left(\mathrm{Cr}^{3+}\right)=-1109.6 \pm 70 \mathrm{kcal} / \mathrm{mol}\right]$ that now matches the experimental value within the uncertainty of this magnitude. The use of the polarizable water model lowers by $\sim 140 \mathrm{kcal} / \mathrm{mol}$ the statistical estimation of the $\left[\mathrm{Cr}\left(\mathrm{H}_{2} \mathrm{O}_{6}\right)\right]^{3+}$ hydration enthalpy compared to the nonpolarizable model. $(-573 \mathrm{kcal} / \mathrm{mol}$ for the polarizable model vs $-714 \mathrm{kcal} / \mathrm{mol}$ for the nonpolarizable one.) This improvement reflects a more accurate treatment of the many-body nonadditive effects. (C) 2000 American Institute of Physics. [S0021-9606(00)50804-6]
\end{abstract}

\section{INTRODUCTION}

Ionic solutions are systems of special interest due to the large number of physicochemical processes they are involved in. Fields ranging from biochemistry to chemical engineering benefit from the detailed understanding of their behavior. ${ }^{1-5}$ Rationalization of their properties has led to the search for microscopical interpretations where the extrathermodynamical assumptions of ionic contributions could be reasonably handled. Among the most popular techniques to address the study of this topic are classical computer simulations, ${ }^{6,7}$ Monte Carlo (MC) and molecular dynamics (MD), which are able to consider the system at a molecular level. However, the success of the results strongly depends, apart from other factors, such as number of particles, boundary conditions, etc., on the interaction potentials used to describe the forces acting among the components of the model system. ${ }^{8}$ The model potentials are usually derived either from experimental data or from quantum-mechanical computations. Our strategy relies on first-principles interaction po-

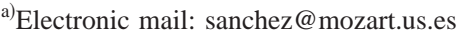

tentials that do not introduce empirical factors into the statistical calculations. ${ }^{9}$ However, this approach implies a high computational cost and its ability to produce results in good agreement with experimental data depends on various factors, especially when complex systems are modeled: the quality of the $a b$ initio calculations, the adequate sampling of the interaction hypersurfaces and the explicit inclusion of nonadditive collective effects. Consideration of these factors and their inclusion in the model potentials should generally improve the modelization and lead to more accurate descriptions of the simulated systems. In the present work we explicitly address this point.

One of the most usual assumptions to describe the particle interactions is that of the pairwise additivity. In the case of highly charged cations immersed in polar solvents, like water, such a premise fails. ${ }^{10}$ Three main aspects should be considered to understand this failure:

(1) The neglect of the nonadditive behavior of the classical electric polarization, ${ }^{11-13}$ and, to a lesser extent, due to exchange contributions and charge transfer phenomena,${ }^{14-16}$ leads to an overestimation of the pair 
binding energy. This problem has been addressed by including three- and four-body terms, as well as the use of polarizable water models, and quite reasonable results have been obtained on singly charged cations. ${ }^{17-24}$ In the case of doubly charged ones, a clear improvement is observed, ${ }^{25-31}$ especially on structural parameters. Furthermore, for several lanthanide ions $\left(\mathrm{Ln}^{3+}\right)$ in aqueous solutions, Kowall et al. ${ }^{32}$ applied a polarizable water model that enables the description of the decrease in the water-exchange rate, $\mathrm{Ln}-\mathrm{O}$ distance ("lanthanide contraction") and the coordination number along the series.

(2) The second problem associated with the development of first-principles ion-water interaction potentials stems from the fact that the correct dissociation limit for a $\left[\mathrm{M}\left(\mathrm{H}_{2} \mathrm{O}\right)\right]^{m+}$ cluster is the charge transfer state $\left[\mathrm{M}^{(m-1)+}+\mathrm{H}_{2} \mathrm{O}^{+}\right]$instead of the expected $\left[\mathrm{M}^{m+}\right.$ $+\mathrm{H}_{2} \mathrm{O}$ ]. This avoided crossing is due to the relative value of the water electron affinity and the $m$ th ionization potential of the metal. ${ }^{33-35}$ Nevertheless, this behavior does not occur for larger complexes; sometimes the addition of a second water molecule is enough and the dissociation limit $\mathrm{H}_{2} \mathrm{O}+\left[\mathrm{M}\left(\mathrm{H}_{2} \mathrm{O}\right)\right]^{m+}$ applies. ${ }^{36}$

(3) Finally, a third aspect has to be considered for the interesting case of transition metal cations. Perturbation on the metal ion induced by the probe water molecule may easily change its electronic state: the set of partially filled $d$ orbitals defines a narrow energy spectrum where several electronic arrangements are defined.

A strategy that overcomes to a great extent the previous difficulties is the use of the hydrated ion, $\left[\mathrm{M}\left(\mathrm{H}_{2} \mathrm{O}\right)_{n}\right]^{m+}$, as the representative entity in solution, instead of the bare ion, $\mathrm{M}^{m+}$. In this case, the bare ion-water interaction potential is replaced by the hydrated ion-water (HIW) one. The Sevilla group has developed $a b$ initio HIW potentials for $\mathrm{Zn}^{2+}$ and for $\mathrm{Cr}^{3+}$ that were used in Monte Carlo simulations to obtain energetic and structural information ${ }^{37-39}$; to study the dynamics, additional molecular dynamics simulations were performed in the latter case. ${ }^{40,41}$ Also, the methodology has recently been extended by proposing a low-cost technique for the generalization of the HIW potential to other small and highly charged cations like $\mathrm{Be}^{2+}, \mathrm{Mg}^{2+}$, and $\mathrm{Al}^{3+}{ }^{42}$ The validity of this approach has also recently been tested by other groups ${ }^{43-46}$ and was pointed out by Cordeiro et al. ${ }^{47}$ in an earlier $\mathrm{Cu}^{2+}$ hydration study. In particular, Bleuzen et $a l .{ }^{43}$ have studied the water-exchange mechanism for the second hydration shell of the $\mathrm{Cr}^{3+}$, assuming in the ionwater interaction potential the existence of the hexahydrate and selecting the nonelectrostatic parameters of the ionsolvent potential in such a way that MD simulations produced the closer results to experimental properties. Wasserman et al. have studied the $\mathrm{Al}^{3+}$ hydration by considering an aluminum-water potential based on the ab initio information (structure and vibrational spectrum) of the $\left[\mathrm{Al}\left(\mathrm{H}_{2} \mathrm{O}\right)_{6}\right]^{3+}$ cluster and using a flexible, but nonpolarizable, water model.

The case of $\mathrm{Cr}^{3+}$ hydration is particularly involved because the development of a first-principles ion-water interaction potential suffers to a large extent all the difficulties previously mentioned. This fact is reflected in the overestimation by $\sim 13 \%(-150 \mathrm{kcal} / \mathrm{mol})$ of the hydration enthalpy $\Delta H_{\text {hydr }}\left(\mathrm{Cr}^{3+}\right)$, obtained from Monte Carlo simulations with a HIW potential for the trivalent ion-water interactions. ${ }^{39}$ Additional quantum-mechanical calculations over a set of clusters led us to conclude that although most of the many-body effects are associated with the first hydration sphere, which is correctly treated by the HIW approach, a non-negligible contribution is still present in the second hydration shell. ${ }^{39} A b$ initio calculations on the $\left[\mathrm{Cr}\left(\mathrm{H}_{2} \mathrm{O}\right)_{6}\right]^{+3}\left(\mathrm{H}_{2} \mathrm{O}\right)_{12}$ cluster showed that almost half of the overestimation (about $65 \mathrm{kcal} / \mathrm{mol}$ ) is due to the many-body effects present in the second hydration shell. In the simulations performed for this cation, water-water interactions were described by nonpolarizable water models: $\mathrm{MCY}^{48}$ and TIP4P. ${ }^{49}$

In principle, a realistic potential should be able to accurately reproduce all the properties of the system being simulated; the validation against the available experimental data is a requirement to assess the reliability of the model in describing properties that have not been determined experimentally, and in providing an explanation in terms of intermolecular interactions. In the case of the hydration of ions, the correct reproduction of energetics is crucial. The nonempirical character of the HIW interaction potential allows its refinement in several ways that can lead to improvements of its predictions. Thus, using a polarizable water model should certainly produce a more accurate description of hydration. For instance, Rustad et al. ${ }^{50}$ combined their own polarizable water model $(\mathrm{HHR})^{51}$ with a $\mathrm{Fe}^{3+}-\mathrm{H}_{2} \mathrm{O}$ interaction potential in their study of the $\mathrm{Fe}^{3+}$ solvation and its hydrolysis products in aqueous solution, obtaining encouraging results and concluding that their model could be improved by considering the hydrated forms of the $\mathrm{Fe}^{3+}$ and its hydrolysis products to build the $a b$ initio interaction potentials. This is precisely the issue in this work: a coupling between the original HIW potential and a model of water that is consistent with it, i.e., built from first-principles, and with the ability to include explicitly nonadditive effects. The water-water interaction potential chosen incorporates the polarizable character of the solvent molecule by means of mobile charges joined to the atoms by harmonic oscillators. This model has been developed by the Cuernavaca group and was called the MCHO potential. ${ }^{52}$ It has proven to produce satisfactory results in modeling the hydration of quite different solutes..$^{25,26,53,54}$ To test the validity of this coupling, a set of cluster computations is performed with different analytical potentials, with the aim of evaluating whether or not the energetic aspects can be improved when the polarizable water model is included without severely affecting other properties of the solution, such as the structural ones that are already fairly described. In a second stage, Monte Carlo simulations are performed to study the effects of the coupling between both potentials when the solvent is fully considered in the $\mathrm{Cr}^{3+}$ hydration description. 


\section{METHODOLOGY}

\section{A. Outline of the HIW and MCHO potentials}

A short overview of these models is presented here to facilitate the understanding of the strategy followed in this work. The details of the HIW and MCHO interaction potentials can be found in the original works, Refs. 39 and 52, respectively.

The basic assumption underlying the HIW model is to consider the water molecules in the first hydration shell distinct from the rest of the solvent molecules, hence permitting a different treatment for their description, the origin of this idea being the high perturbation induced by the cation on the closest solvent molecules. ${ }^{55}$ In the particular case of $\mathrm{Cr}^{3+}$, there are six water molecules in the first hydration shell and they have a mean residence time on the order of 24 hours. ${ }^{56}$ This allows us to consider the interaction potential between the $\left[\mathrm{Cr}\left(\mathrm{H}_{2} \mathrm{O}\right)_{6}\right]^{3+}$ unit and a water molecule, i.e., the HIW potential, in computer statistical simulations. The development of the HIW model starts by optimizing the geometry of the hydrate using an ab initio method, followed by a sampling of the $\left[\mathrm{M}\left(\mathrm{H}_{2} \mathrm{O}\right)_{n}\right]^{m+}-\mathrm{H}_{2} \mathrm{O}$ potential energy surface. It is worth pointing out that the $\left[\mathrm{Cr}\left(\mathrm{H}_{2} \mathrm{O}\right)_{6}\right]^{3+}$ geometry used to extract the interaction potential and for further statistical simulations is that obtained by ab initio computations. Thus, the geometrical structure of the first-shell water molecules $\left(d_{\mathrm{O}-\mathrm{H}}=0.967 \AA,<\mathrm{HOH}=107.54^{\circ}\right)$ is different from the rest of the water molecules of the bulk $\left(d_{\mathrm{O}-\mathrm{H}}=0.957 \AA\right.$, $\left.<\mathrm{HOH}=104.5^{\circ}\right)$. The structural complexity of the hydrate, unlike monoatomic ions, forces the evaluation of a large number $(\sim 1200)$ of quantum-chemical structures of the hydrate and the probe water molecule. The ab initio pair interaction energies are then fitted to an analytical function built on the basis of site-site distances, $r_{i j}$,

$$
E_{\mathrm{HIW}}=\sum_{i}^{\mathrm{HI}} \sum_{j} \frac{C_{4}^{i j}}{r_{i j}^{4}}+\frac{C_{6}^{i j}}{r_{i j}^{6}}+\frac{C_{12}^{i j}}{r_{i j}^{12}}+\frac{q_{i} q_{j}}{r_{i j}} .
$$

Two contributions may be distinguished in the previous equation. The first one accounts for the short-range interactions and is described by a linear combination of $r^{-n}$ $(n>3)$ terms. The second contribution is Coulombic; the charges on the water molecule are those of the model employed, and for the hydrate they are derived from a fitting procedure $^{57}$ to reproduce the ab initio molecular electrostatic potential of the hydrate wave function polarized by a dielectric continuum, in order to include the bulk solvent effects in an averaged manner.

It is again convenient to underline how the first-shell water molecules differ from the rest of water molecules in their charge distribution. Thus, they support a partial charge transfer from the cation so that these water molecules are not electrically neutral $(+0.091 e)$ as bulk water molecules are. The significant difference between the first-shell water molecules and bulk ones can be illustrated by comparing the results of the optimization of two water dimers. One of them is formed by two TIP4P water molecules and the other one by a TIP4P water and a water molecule of the first shell of the hydrate. Interaction energies are $-26.1 \mathrm{~kJ} / \mathrm{mol}$ for $\left(\mathrm{H}_{2} \mathrm{O}\right)_{\text {TIP4P }}-\left(\mathrm{H}_{2} \mathrm{O}\right)_{\text {TIP } 4 \mathrm{P}}$ and $-82.5 \mathrm{~kJ} / \mathrm{mol}$ for

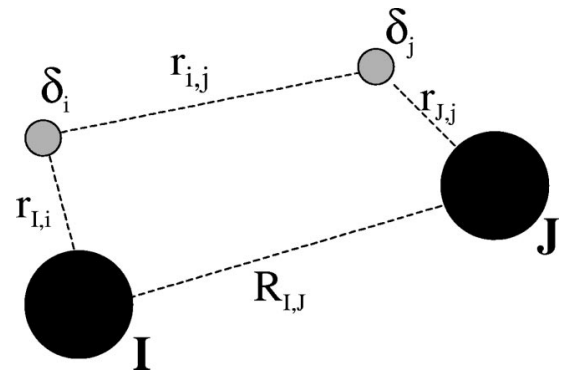

FIG. 1. Picture describing the MCHO model. $I$ and $J$ correspond to the atomic nuclei, and $\delta_{i}$ and $\delta_{j}$ to their respective mobile net charges.

$\left(\mathrm{H}_{2} \mathrm{O}\right)_{1 \text { st-shell }}-\left(\mathrm{H}_{2} \mathrm{O}\right)_{\mathrm{TIP} 4 \mathrm{P}} ;$ and $\mathrm{R}_{\mathrm{O}-\mathrm{O}}$ is $2.75 \AA$ for $\left(\mathrm{H}_{2} \mathrm{O}\right)_{\text {TIP4P }}-\left(\mathrm{H}_{2} \mathrm{O}\right)_{\text {TIP4P }}$ and $2.55 \AA$ for $\left(\mathrm{H}_{2} \mathrm{O}\right)_{1 \text { st-shell }}$ $-\left(\mathrm{H}_{2} \mathrm{O}\right)_{\text {TIP4P }}$. As expected, the first-second shell water interaction is much stronger than the water-water interaction in the bulk.

Among the possible polarizable water models, ${ }^{8,19,22,32,51,59,58-60}$ the MCHO potential was chosen mainly because the underlying philosophy of its building and the geometry of the water molecule are completely consistent with the perspective used in the development of the HIW model. The information source is a set of quantummechanical computations and the $C_{L}(L=4,6,12)$ coefficients of expression (1) were obtained using the rigid MCY water model, which has exactly the same nuclear relative positions as MCHO, thus facilitating the coupling with the HIW potential. The MCHO water model is of atom-atom type, each atom having ascribed a center $I$ and a net mobile charge $\delta_{i}$ (Fig. 1). The expression for the atom-atom potential is

$$
\begin{aligned}
E_{i, j}^{\mathrm{MCHO}}= & A_{i, j} e^{-n_{1} R_{I, J}}+B_{i, j} e^{-n_{2} R_{I, J}}+C \frac{\delta_{i} \delta_{j}}{r_{i, j}} \\
& +a_{i} r_{I, i}^{2}+a_{j} r_{J, j}^{2}+\frac{k}{r_{i, j}^{n_{3}}},
\end{aligned}
$$

where subscripts' capital letters denote nuclear centers and subscripts' noncapital letters denote net charges. The first two terms define the short-range contribution, not appearing in intramolecular interactions, i.e., no vibrations are allowed. $C$ is equal to 1 for intermolecular interactions, and in the case of intramolecular ones, its value depends on the $i-j$ pair. The $a_{i}$ parameters are half the force constants corresponding to the harmonic oscillator behavior adopted for the interaction of a given nucleus $I$ with its respective net mobile electric charge $\delta_{i}$. The last term in Eq. (2) avoids the collapse of opposite sign charges. The parameters were fitted to the MCY pair interaction surface, and to ab initio computed surfaces of three- and four-body nonadditive contributions to the interaction energy. Resembling the Born-Oppenheimer approximation, the mobile charges adjust their positions to the local electric field for a given configuration of the nuclear centers. Thus whereas the $R_{I, J}$ distances are directly obtained from nuclear positions, the $r_{i, j}$ distances can only be computed after the positions of the mobile net charges have been obtained from solving the zero force equations by means of an iterative, self-consistent procedure. The result is a model 
TABLE I. Fitted values for the $k$ and $n$ parameters. $\mathrm{O}_{\mathrm{I}}$ and $\mathrm{H}_{\mathrm{I}}$ denote centers belonging to the hydrated ion. When values of the table are replaced in the modified Eq. (1), energy is obtained in $\mathrm{kcal} / \mathrm{mol}$ if distances and charges are given in a.u.

\begin{tabular}{ccc}
\hline \hline Pair & $k$ & $n$ \\
\hline $\mathrm{O}_{\mathrm{I}}-\mathrm{H}$ & 6375.9349 & 9.805506 \\
$\mathrm{H}_{\mathrm{I}}-\mathrm{O}$ & 196.87962 & 3.278571 \\
\hline \hline
\end{tabular}

capable of including all the many-body nonadditive effects in the system; it was explicitly adjusted up to only fourth order because usually the contributions of higher order to the intermolecular interaction are negligible. Therefore a site-site formalism is kept that is simple enough to be effectively used in numerical simulations.

\section{B. Development of the hydrated ion-water polarizable potential}

The simplest strategy to couple the HIW and the MCHO models would be their simple addition, that is, to use Eqs. (1) and (2) as they are, to compute ion-water and water-water interactions. However, this approach is bound to fail due to two different and independent reasons. The first one is related to the polarization of the water molecule interacting with the hexahydrate. When the original HIW was developed, the use of a nonpolarizable water model forced the fitting to include the polarization effects on the $C_{L}$ coefficients in Eq. (1) in an averaged manner, because these effects are present in the interaction energies obtained from $a b$ initio computations. On the other hand, the mobile charges on the MCHO model explicitly include the induction effects on the Coulombic part. In this sense, the nonadditive contributions to the interaction energy would be counted twice by the simple addition of both models. The second factor against this strategy is the excessive polarization induced on the MCHO waters produced by the lack of polarizability of the hydrate, thus causing an exaggerated reorganization of the mobile charges. This effect is further enhanced by the lack of a repulsive term in the interactions for charges of different sign between the $\mathrm{HI}$ and the $\mathrm{MCHO}$ water, unlike what happens in the MCHO model [last term of Eq. (2)]. Both factors lead to an exaggerated HI-MCHO water interaction. Kowall et ll $^{32}$ have found the increase (more negative values) of the solvation energy for a set of lanthanide ions in water when an unpolarizable water model is changed by a polarizable one.

Once the failure of the simplest strategy has been rationalized, different options to perform the coupling come up. In this work, a special effort is done to keep the short-range terms of the HIW potential unaltered, due to the important role played by a correct description of the van der Waals envelope. ${ }^{61}$ Bearing in mind this point and taking into account the terms describing the water-water interactions, one of the easiest options by which to proceed is by adding to the original HIW expression a repulsive term between the electric charges, in the same way as in the MCHO model, that is, a new term of the type $k / r^{n}$. The $k$ and $n$ parameters are then fitted using the original single point calculations of the

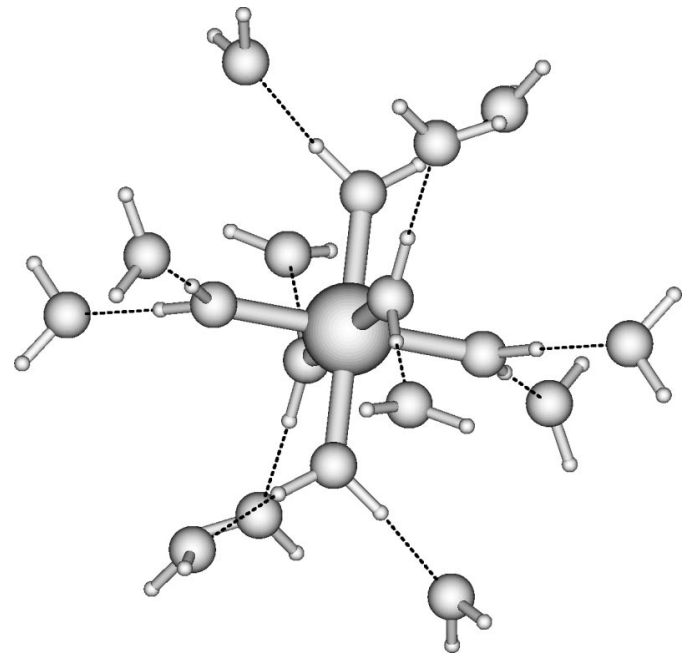

FIG. 2. Structure of the minimum corresponding to 12 water molecules interacting with the $\mathrm{Cr}^{3+}$ hydrate using the $\mathrm{HIW}_{P}$ and the MCHO potentials. Drawn bonds between the cation and oxygens of the first-shell water molecules represent the feature of the $\mathrm{HIW}_{P}$ to consider the hydrate as a single unit.

$\left[\mathrm{Cr}\left(\mathrm{H}_{2} \mathrm{O}\right)_{6}\right]^{3+}-\mathrm{H}_{2} \mathrm{O}$ ab initio surface. Table I collects the fitted parameters. Henceforth this new potential will be called $\mathrm{HIW}_{P}$ (the subscript ' $P$ ', denotes that this HIW potential was set up to be used with a Polarizable water model). The success of this strategy will be discussed on the basis of the results presented in the next section.

\section{RESULTS AND DISCUSSION}

\section{A. Many-body effects in the $\mathrm{Cr}^{3+}$ second hydration shell}

As already pointed out in Sec. I, previous studies have shown that for systems formed by ions and polar solvents, such as water, the nonadditivity of the interactions is particularly important in the closest environment of the ion. When the hydrated ion approach is used, the many-body terms within the first hydration shell are implicitly taken into account by the quantum-mechanical computations; but beyond this shell the trivalent cation field can still produce large cooperative phenomena in polar solvents. The first neighbors explicitly considered in the simulations using the HIW potential are those of the second hydration sphere, so it is of interest to examine to what extent the inclusion of the polarizable water model and the new fitting of the HIW potential are able to deal with the remaining many-body contributions of this region. To obtain this information, a cluster formed by the hydrate and a second hydration with 12 water molecules, $\left[\mathrm{Cr}\left(\mathrm{H}_{2} \mathrm{O}\right)_{6}\right]^{3+}\left(\mathrm{H}_{2} \mathrm{O}\right)_{12}$, has been optimized by using the $\mathrm{HIW}_{P}+\mathrm{MCHO}$ interaction potentials. Figure 2 shows the optimized geometry of this cluster. A quite symmetric arrangement around the hexahydrate is obtained, where the second-shell water molecules bind by pairs to each one of the first-shell water molecules. The optimized geometrical parameters, the total interaction energy, $\Delta E_{\text {int }}$ (total), and its decomposition in hydrated ion-water (HI-W) and water- 
TABLE II. Interaction energy, $\Delta E_{\text {int }}$, between the $\mathrm{Cr}^{3+}$ hexahydrate and its hydration shell, $\left[\mathrm{Cr}\left(\mathrm{H}_{2} \mathrm{O}\right)_{6}\right]^{+3} \cdot\left(\mathrm{H}_{2} \mathrm{O}\right)_{12}$ computed from different couples of analytical interaction potentials and ab initio methods, and its decomposition in hydrated ion-water $(\mathrm{HI}-\mathrm{W})$ and water-water $(\mathrm{W}-\mathrm{W})$ contributions. $\Sigma E_{2-\text { body }}$ is the $a b$ initio interaction energy assuming pairwise additivity. $R\left(\mathrm{Cr}-\mathrm{O}_{\mathrm{II}}\right)$ and $R\left(\mathrm{Cr}-\mathrm{H}_{\mathrm{II}}\right)$ are the optimized distances using the different analytical potentials. (Energies in $\mathrm{kcal} / \mathrm{mol}$ and distances in $\AA$.)

\begin{tabular}{lccccc}
\hline \hline \multicolumn{3}{c}{ Analytical potentials } & \multicolumn{2}{c}{ ab initio } \\
\hline Interaction energy & $\mathrm{HIW}_{P}+\mathrm{MCHO}$ & $\mathrm{HIW}+\mathrm{MCY}$ & $\mathrm{HIW}+\mathrm{TIP} 4 \mathrm{P}$ & & \\
& $\Delta E_{\text {int }}$ & $\Delta E_{\text {int }}$ & $\Delta E_{\text {int }}$ & $\Sigma E_{\text {2-body }}$ & -396.1 \\
total & -365.9 & -394.4 & -387.9 & -328.9 & -459.2 \\
$\mathrm{HI}-\mathrm{W}$ & -433.7 & -438.5 & -428.2 & -388.3 & 63.1 \\
$\mathrm{~W}-\mathrm{W}$ & 67.8 & 44.1 & 40.3 & 59.4 & \\
Geometrical parameters & & & & $4.15^{\mathrm{a}}$ & $4.15^{\mathrm{a}}$ \\
$R\left(\mathrm{Cr}-\mathrm{O}_{\mathrm{II}}\right)$ & 4.21 & 4.15 & 4.17 & $4.80^{\mathrm{a}}$ & $4.80^{\mathrm{a}}$ \\
$R\left(\mathrm{Cr}-\mathrm{H}_{\mathrm{II}}\right)$ & 4.86 & 4.80 & 4.82 & & \\
\hline \hline
\end{tabular}

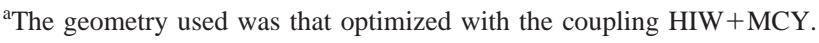

water $(\mathrm{W}-\mathrm{W})$ contributions are given in Table II. The polarizable character of the water molecules forces the evaluation of these two contributions retaining the polarization acquired in the whole cluster. For the sake of comparison with previous unpolarizable models, geometries obtained for the $\left[\mathrm{Cr}\left(\mathrm{H}_{2} \mathrm{O}\right)_{6}\right]^{3+}\left(\mathrm{H}_{2} \mathrm{O}\right)_{12}$ cluster with the previous couples of potentials, $\mathrm{HIW}+\mathrm{MCY}^{39}$ and $\mathrm{HIW}+\mathrm{TIP} 4 \mathrm{P},{ }^{40}$ have also been included.

Likewise, the ab initio energies corresponding to the interaction between the hydrate and the second shell of water molecules, $\Delta E_{\text {int }}$, and that obtained under the assumption of pairwise additivity among the hydrate and the 12 water molecules, $\Sigma E_{2 \text {-body }}$, have been included in Table II. These results were already obtained to test the behavior of the original $\mathrm{Cr}^{3+}$ HIW potential that was coupled with the MCY water potential. ${ }^{39}$ The geometry used for the $a b$ initio quantum-chemical calculations was that obtained by the optimization of the cluster with the HIW + MCY potentials. The level of calculation is the same as that used to generate the potential energy surface which fitted the original HIW potential. $^{39}$ As usual, the total interaction energy, $\Delta E_{\text {int }}$ (total), was computed as the difference between the energy of the whole cluster and that of the components

$$
\begin{aligned}
\Delta E_{\text {int }}(\text { total })= & E\left(\left[\mathrm{Cr}\left(\mathrm{H}_{2} \mathrm{O}\right)_{6}\right]^{3+}\left(\mathrm{H}_{2} \mathrm{O}\right)_{12}\right) \\
& -E\left(\left[\mathrm{Cr}\left(\mathrm{H}_{2} \mathrm{O}\right)_{6}\right]^{3+}\right)-12 E\left(\mathrm{H}_{2} \mathrm{O}\right) \\
= & \Delta E(\mathrm{HI}-\mathrm{W})+\Delta E(\mathrm{~W}-\mathrm{W}),
\end{aligned}
$$

being $\Delta E(\mathrm{~W}-\mathrm{W})=E\left(\left[\mathrm{H}_{2} \mathrm{O}\right]_{12}\right)-12 E\left(\mathrm{H}_{2} \mathrm{O}\right)$, and $\Delta E(\mathrm{HI}-\mathrm{W})$ being obtained by difference. For the calculation of $\Sigma E_{2 \text {-body }}$, the ab initio interaction energy is computed on the basis of the pairwise additivity approximation. However, for clarity reasons it is convenient to underline that the hydrated ion is still considered as a unique entity. As a consequence, any pair interaction involving the hydrate contains implicitly interactions with seven molecular species, the $\mathrm{Cr}^{3+}$ cation and six highly perturbed water molecules. Due to the symmetry of the structure, the 12 hydrated ion-water interactions were considered equivalent,

$$
\begin{aligned}
\Sigma E_{2 \text {-body }}(\text { total })= & 12\left\{E\left(\left[\mathrm{Cr}\left(\mathrm{H}_{2} \mathrm{O}\right)_{6}\right]^{3+}\left(\mathrm{H}_{2} \mathrm{O}\right)\right)\right. \\
& \left.-E\left(\left[\mathrm{Cr}\left(\mathrm{H}_{2} \mathrm{O}\right)_{6}\right]^{3+}\right)-E\left(\mathrm{H}_{2} \mathrm{O}\right)\right\} \\
& +\sum_{i=1}^{12} \sum_{j>i}^{12}\left\{E\left(\mathrm{H}_{2} \mathrm{O}\right)_{i}\left(\mathrm{H}_{2} \mathrm{O}\right)_{j}-2 E\left(\mathrm{H}_{2} \mathrm{O}\right)\right\} .
\end{aligned}
$$

Comparison of the two $a b$ initio total interaction energies (Table II) shows a many-body correction of $67.2 \mathrm{kcal} /$ mol $\left[\Delta E_{\text {int }}\right.$ (total) $-\Sigma E_{2 \text {-body }}$ (total) $]$. To get insight into the origin of this amount some decomposition has to be envisaged. The self-consistent character of the quantummechanical computations leads to the definition of a given partition scheme for $\Delta E_{\text {int }}$ (total) that partially clouds the actual contributions in the whole cluster. That is, according to Eq. (3), $\Delta E_{\text {int }}(\mathrm{W}-\mathrm{W})$ is computed for a cluster of water molecules that is not being polarized by the hydrated cation, therefore the value included in Table II $(59.4 \mathrm{kcal} / \mathrm{mol})$ is a lower limit to the water-water contribution in the whole cluster. As a consequence, the hydrated ion-water interaction, which is obtained by subtracting the water-water contribution to the total interaction energy $\left[\Delta E_{\mathrm{int}}(\mathrm{HI}-\mathrm{W})\right.$ $=\Delta E_{\text {int }}($ total $\left.)-\Delta E_{\text {int }}(\mathrm{W}-\mathrm{W})\right]$, is an upper limit to this contribution ("exact" $E_{\mathrm{HI}-\mathrm{W}}<-388.3 \mathrm{kcal} / \mathrm{mol}$ ). The same reasoning can be applied to the decomposition in the case of the two-body approach, $\Sigma E_{2 \text {-body }}$, but it should be noted that water-water repulsions should be less affected by not including the ionic polarization, given that the considered structures only involved two water molecules. As already pointed out by Probst ${ }^{62}$ and Curtiss et al. ${ }^{13}$ it is observed that many-body contributions are more important in the hydrated ion-water interactions than in the water-water ones.

Comparison of the $a b$ initio energies with the results derived from the application of the analytical potentials emphasizes two points: the first one concerns the total interaction energies and the second its components. As expected, the inclusion of polarization in water molecules with the $\mathrm{HIW}_{P}-\mathrm{MCHO}$ coupled potentials accounts for a part of the many-body terms reducing the difference with respect to the ab initio $\Delta E_{\text {int }}$ (total) to only $37 \mathrm{kcal} / \mathrm{mol}$. Therefore, the ref- 
erence is not any longer the two-body results, $\Sigma E_{2 \text {-body }}$ (total), as it is the case for the two previous couples of potentials HIW + MCY and HIW+TIP4P. Regarding the second point, the value for the hydrated ion-water interaction energies computed by means of the $\mathrm{HIW}_{P}-\mathrm{MCHO}$ potentials is similar to the previous values obtained from the HIW + MCY and HIW + TIP4P potentials, given that the new hydrate-water potential $\mathrm{HIW}_{P}$ fits the same set of $a b$ initio points. However, the MCHO water-water potential describes a more repulsive situation among water molecules in the second hydration shell than that obtained by the nonpolarizable MCY and TIP4P models. This leads to a better value for water-water repulsions given that for this computation $(67.8 \mathrm{kcal} / \mathrm{mol})$ the second hydration shell has retained the polarization induced by the hydrate. It is clear that $a b$ initio values are lower limits of the actual water-water repulsions in the whole cluster ["exact", $\left.\Delta E_{\text {int }}(\mathrm{W}-\mathrm{W})>59.4 \mathrm{kcal} / \mathrm{mol}\right]$. On the contrary, the waterwater interaction energies computed by the TIP4P and MCY water models are less repulsive than the $a b$ initio value by about $20 \mathrm{kcal} / \mathrm{mol}$. However, a fortuitous (and favorable) partial cancellation with the corresponding HIW potentials leads to total interaction energies close to the $\Sigma E_{2 \text {-body }}($ total) in these two cases. Therefore, it may be concluded that the new couple of interaction potentials, $\mathrm{HIW}_{P}+\mathrm{MCHO}$, is able to improve the estimation of the total interaction energy of the $\mathrm{Cr}^{3+}$ hexahydrate with its hydration shell, by supplying a more equilibrated description of the hydrated ion-water and water-water interactions.

Possible origins of the discrepancy of $37 \mathrm{kcal} / \mathrm{mol}$ in $\Delta E_{\text {int }}\left(\right.$ total) between the ab initio and $\mathrm{HIW}_{P}+\mathrm{MCHO}$ values must be mentioned to get a more complete view of the reliability of the developed potential. First of all, the basis sets used for the quantum chemical computations of the HIW potential energy surface are different from those used to build the MCHO potential. Second, some many-body interactions have not been included in the parametrization, particularly three-body water-HI-water and four-body waterwater-HI-water contributions. The lack of instantaneous polarization of the hydrate is partially the origin of this limitation. Finally, the intrinsic error associated with the fitting of the interaction energy by the potentials employed must also contribute to the discrepancy.

The trend observed in $R\left(\mathrm{Cr}-\mathrm{O}_{\mathrm{II}}\right)$ and $R\left(\mathrm{Cr}-\mathrm{H}_{\mathrm{II}}\right)$ is easily understood on the basis of the inclusion of the polarizable character for water molecules. If the same hydrated ionwater potential is retained, the water molecule at the second shell will be polarized mainly by its interaction with the triply charged hydrate and the repulsion among solvent molecules (due to dipole-dipole interactions) leads to larger distances from the cation, e.g., $4.21 \AA$ for $\mathrm{Cr}-\mathrm{O}_{\mathrm{II}}$ distance, than those previously observed for the nonpolarizable water models, MCY (4.15 ̊) and TIP4P (4.17 $\AA$ ).

\section{B. Monte Carlo simulations}

MC simulation conditions were the same as in the previous work ${ }^{39}$ in order to make the comparison as direct as possible. Thus, the system was formed by one $\left[\mathrm{Cr}\left(\mathrm{H}_{2} \mathrm{O}\right)_{6}\right]^{3+}$ cation and 512 water molecules. Numerical simulations have

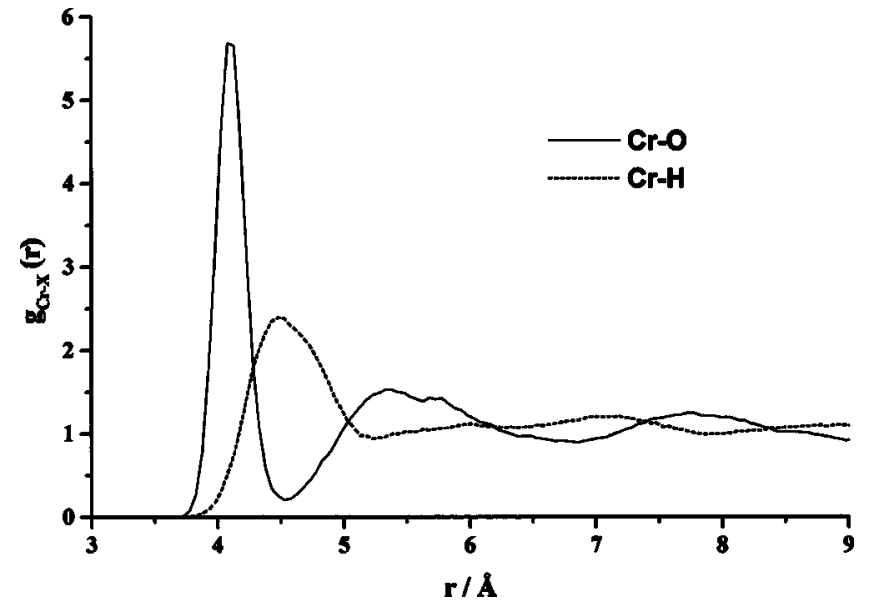

FIG. 3. $\mathrm{Cr}-\mathrm{O}$ (solid line) and $\mathrm{Cr}-\mathrm{H}$ (dashed line) radial distribution functions for the Monte Carlo simulation of the $\left[\mathrm{Cr}\left(\mathrm{H}_{2} \mathrm{O}\right)_{6}\right]^{3+}$ with $512 \mathrm{H}_{2} \mathrm{O}$.

been performed under periodic boundary conditions in the NVT ensemble using the algorithm of Metropolis et al. ${ }^{63}$ as implemented in the MONTECUERNA ${ }^{64}$ code. A cubic box of $24.8 \AA$ was used in both cases. The program, which uses the MCHO water model, has been modified to include the $\mathrm{HIW}_{P}$ potential and the Ewald sum technique ${ }^{6,7}$ for the evaluation of Coulomb interactions. For the simulation containing the ion, the Ewald sum procedure included the charge system term. ${ }^{65,66}$ A spherical molecular cutoff of $L / 2$ was applied to the real space part of the Ewald energy as well as the shortrange potentials. The system was equilibrated at $298 \mathrm{~K}$ with 20 million configurations and 40 million configurations were additionally produced for statistical analysis. The pure solvent reference was obtained from a $512 \mathrm{MCHO}$ water molecules simulation under the same conditions. Equilibration and production runs were obtained with 20 and 34 million configurations, respectively.

The resulting radial distribution functions (RDF) for the $\mathrm{Cr}-\mathrm{O}$ and $\mathrm{Cr}-\mathrm{H}$ pairs have been plotted in Fig. 3. Obviously, since our modeling of the ionic solution considers the $\left[\mathrm{Cr}\left(\mathrm{H}_{2} \mathrm{O}\right)_{6}\right]^{3+}$ cluster as a single cationic entity, the structural information obtained is concerned with the second hydration shell onward. The $\mathrm{Cr}-\mathrm{O}$ RDF shows a well-defined peak centered at $4.08 \AA$ followed by a significant depletion zone with a minimum at $4.5 \AA$. The integration of this peak leads to $12.3 \pm 0.1$ water molecules. The $\mathrm{Cr}-\mathrm{H}$ RDF confirms the presence of a well-defined second hydration shell; a peak centered at $4.48 \AA$ is the main characteristic of this function. However, this peak is about three times broader than the one corresponding to the $\mathrm{Cr}-\mathrm{O} \mathrm{RDF}$. This reflects the permitted motions of water molecules in the second shell: those involving rotational and librational modes, whereas those related to large changes of $\mathrm{Cr}-\mathrm{O}_{\mathrm{II}}$ distances are highly restricted. Previous dynamics results with a nonpolarizable water model support this finding. ${ }^{40,41}$ Comparison of these two RDFs with those obtained by the application of the HIW potential and nonpolarizable water models, such as $\mathrm{MCY}^{39}$ and TIP4P, ${ }^{40}$ shows that although the main features of the curves are reproduced, some differences are worth being pointed out. There is a shortening in the $\mathrm{Cr}-\mathrm{O}_{\mathrm{II}}$ distance when going from the isolated clusters, $\left[\mathrm{Cr}\left(\mathrm{H}_{2} \mathrm{O}\right)_{6}\right]^{3+}\left(\mathrm{H}_{2} \mathrm{O}\right)_{12}$, to the 
TABLE III. Average dipole moment of water molecules in different spherical regions around the hexahydrate and in pure water.

\begin{tabular}{lll}
\hline \hline Type of water molecule & Spherical region & $|\boldsymbol{\mu}|$ (Debye) \\
\hline 2nd shell & $r_{\mathrm{CrO}}<4.6 \AA$ & $3.20 \pm 0.03$ \\
3rd shell & $4.6 \leqslant r_{\mathrm{CrO}} \leqslant 6.5 \AA$ & $2.93 \pm 0.02$ \\
Bulk & $r_{\mathrm{CrO}}>6.5 \AA$ & $2.89 \pm 0.01$ \\
Pure & $\cdots$ & $2.89 \pm 0.01$ \\
\hline \hline
\end{tabular}

maxima in the peak of the corresponding $\mathrm{Cr}-\mathrm{O}$ RDFs. This shortening is more pronounced for the $\mathrm{HIW}_{P}-\mathrm{MCHO}$ case $(0.13 \AA)$ than for the HIW-TIP4P $(0.10 \AA)$ or HIW-MCY $(0.09 \AA)$ cases. This can be ascribed to the additional polarization effects induced on the second hydration shell molecules by the third hydration shell. A similar effect, associated with specific interactions between first and second hydration shells, has already been observed in two quantumchemical studies on the hydration of several monoatomic cations. ${ }^{67,68}$

Another difference coming from the inclusion of actual polarization for the water molecules is that the minimum of the $\mathrm{Cr}-\mathrm{O} \mathrm{RDF}$ after the second hydration shell is closer to zero for the polarizable model than for the nonpolarizable one (see Fig. 3). This can be interpreted as a result of a tighter bound shell around the hydrate, which becomes more isolated from the rest of the solution. This is a consequence of the polarization induced on the water molecules of the second shell by the close presence of a highly charged hexahydrate. The same effect can also explain the presence of a broad, but quite defined, shell in the $\mathrm{Cr}-\mathrm{O}$ RDF going from $\sim 5.0$ to $5.8 \AA$ that suggests a third hydration sphere. In short, the actual polarizable water model is favoring the concentric shell model of dilute ionic aqueous solutions, as invoked by Franks and Evans. ${ }^{69}$ In ionic aqueous solutions containing highly charged cations, the ion-solvent interactions are clearly dominant in a widespread region around the cation, determining a large part of the polarization effects on the water molecules. ${ }^{13,39,62}$ Because the dipole moment of the MCHO model responds to polarization, we can look into the value of this magnitude at different distances from the hexahydrate (Table III). The dipole of the second-shell waters is much larger than the dipole of bulk waters, reflecting the strong ionic field to which the former molecules are subject. It is also worthwhile noticing that in spite of the large induced dipole on second-shell waters, the third-shell molecules recover a value close to that of the bulk, showing the well-known buffering capacity of water. ${ }^{70}$ The change of dipole moment value can be seen as a kind of labeling of water molecules following concentric shells of similar degree of electric field perturbation.

An additional interesting parameter to be examined in these systems is the mean distance between oxygen atoms of the first $\left(\mathrm{O}_{\mathrm{I}}\right)$ and outer $(\mathrm{O})$ hydration shells. The corresponding RDF reflects how different the interaction between the waters of the first and second hydration shells is with respect to the typical interaction among water molecules either in pure water or in the bulk of the ionic solution. Figure 4 shows the $\mathrm{O}_{\mathrm{I}}-\mathrm{O}$ RDF of the ionic solution and the $\mathrm{O}-\mathrm{O}$ for

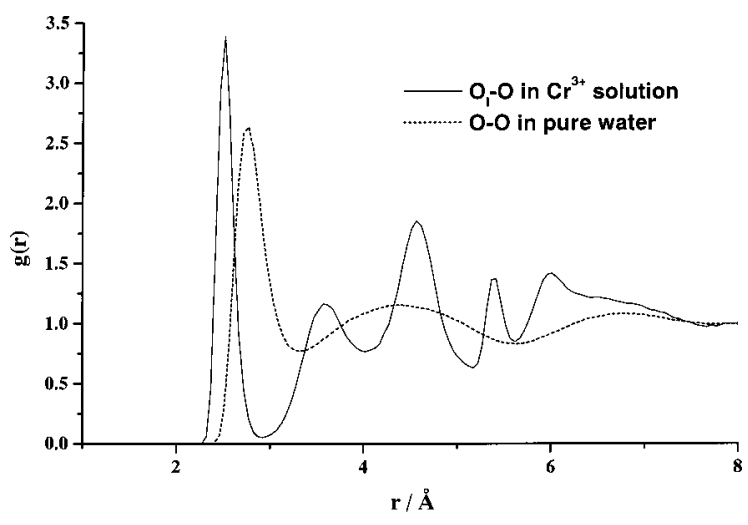

FIG. 4. Oxygen of the hydrate $\left(\mathrm{O}_{I}\right)$-oxygen of the solvent water molecules (O) radial distribution function of the $\left[\mathrm{Cr}\left(\mathrm{H}_{2} \mathrm{O}\right)_{6}\right]^{3+}+512 \mathrm{H}_{2} \mathrm{O}$ simulation (solid line), and $\mathrm{O}-\mathrm{O}$ radial distribution function of the pure water (dashed line).

the pure water simulation with the same water potential. The first peak of the $\mathrm{O}_{\mathrm{I}}-\mathrm{O}$ RDF appears shifted toward shorter values $(\sim 0.25 \AA)$ with respect to the $\mathrm{O}-\mathrm{O}$ RDF of pure water. All these structural results compare well with spectroscopic measurements of several $\mathrm{Cr}(\mathrm{III})$ aqueous solutions obtained by large-angle $\mathrm{x}$-ray scattering (LAXS), ${ }^{71} \mathrm{x}$-ray diffraction (XRD), ${ }^{72}$ neutron diffraction (ND) ${ }^{73}$ extended x-ray absorption fine structure (EXAFS) ${ }^{74}$ and the infrared absorption double-difference technique. ${ }^{75}$

A final comment concerning the structure is the influence that the Ewald summation treatment has on the pure MCHO water simulation. O-O, $\mathrm{H}-\mathrm{O}$ and $\mathrm{H}-\mathrm{H}$ RDFs derived from the simulation with 512 molecules using the Ewald treatment have been compared with those corresponding to a previous simulation ${ }^{52}$ with 343 molecules that did not employ this treatment for long-range interactions. The functions match each other, in agreement with other comparative studies in which it was concluded that the Ewald treatment does not change significantly the atomic paircorrelation functions of polar liquid. ${ }^{66}$

Besides structure, another important point to be examined in this work is the hydration enthalpy of $\mathrm{Cr}^{3+}$, $\Delta H_{\text {hydr }}\left(\mathrm{Cr}^{3+}\right)$, when a polarizable water model is used. The hydrated ion approach allows the statistical evaluation of the hydration enthalpy of the hexahydrate, as the difference between the average energies of the simulations containing the hydrated ion and the pure solvent, that is,

$$
\begin{aligned}
& \Delta H_{\text {hydr }}\left(\left[\mathrm{Cr}\left(\mathrm{H}_{2} \mathrm{O}\right)_{6}\right]^{3+}\right) \\
& \quad=\left\langle E_{\mathrm{HI}}\right\rangle-\left\langle E_{\text {water }}\right\rangle \\
& \quad=(-5596 \pm 25)-(-5023 \pm 20) \mathrm{kcal} / \mathrm{mol} \\
& \quad=-573 \pm 45 \mathrm{kcal} / \mathrm{mol} .
\end{aligned}
$$

Due to the use of the same volume for the pure water and the solution, there is an additional term $\Delta(P V)$ corresponding to the density change in the system when the hydrate is included. Nevertheless, this term can be evaluated and leads to a change of the density value small enough $(\sim 6$ $\times 10^{-3} \mathrm{~g} \mathrm{~cm}^{-3}$ ) to consider this contribution to the $\Delta H_{\text {hydr }}\left(\mathrm{Cr}^{3+}\right)$ negligible. 
To estimate $\Delta H_{\text {hydr }}\left(\mathrm{Cr}^{3+}\right)$, two additional contributions have to be considered: (a) the energy to transfer six water molecules from the pure liquid water to the gas phase, a magnitude usually identified with the vaporization enthalpy $\Delta H_{\text {vap }}$. In our model this quantity is the average waterwater interaction energy of the pure water simulation with opposite sign [from Eq. (5) $\left.-\left\langle E_{\text {water }}\right\rangle / 512=9.8 \mathrm{kcal} / \mathrm{mol}\right]$. (b) The second contribution is the formation enthalpy of the hydrate at $298 \mathrm{~K}, \Delta H_{\text {form }}\left(\left[\mathrm{Cr}\left(\mathrm{H}_{2} \mathrm{O}\right)_{6}\right]^{3+}\right)$, which has already been quantum mechanically computed including the zero point energy and thermal corrections, ${ }^{39}$ therefore,

$$
\begin{aligned}
\Delta H_{\text {hydr }} & \left(\mathrm{Cr}^{3+}\right) \\
= & \Delta H_{\text {hydr }}\left(\left[\mathrm{Cr}\left(\mathrm{H}_{2} \mathrm{O}\right)_{6}\right]^{3+}\right)+6 \Delta H_{\text {vap }} \\
& +\Delta H_{\text {form }}\left(\left[\mathrm{Cr}\left(\mathrm{H}_{2} \mathrm{O}\right)_{6}\right]^{3+}\right) \\
= & -573+58.8-595.4 \mathrm{kcal} / \mathrm{mol}=-1109.6 \mathrm{kcal} / \mathrm{mol} .
\end{aligned}
$$

The experimental estimation of the hydration enthalpy of $\mathrm{Cr}^{3+}$ is $-1116 \mathrm{kcal} / \mathrm{mol}^{76}$ As shown in Eq. (6), the value computed with the polarizable water model agrees pretty well with the experimental estimation. Nevertheless, such a close agreement must be regarded with some caution. We can estimate an uncertainty of at least $\pm 70 \mathrm{kcal} / \mathrm{mol}$ due to the model employed $(45 \mathrm{kcal} / \mathrm{mol}$ derived from the statistical estimate of the $\left[\mathrm{Cr}\left(\mathrm{H}_{2} \mathrm{O}\right)_{6}\right]^{3+}$ enthalpy and $25 \mathrm{kcal} / \mathrm{mol} \mathrm{de}-$ rived from the quantum-chemical estimation of the $\mathrm{Cr}^{3+}$ hexahydrate formation enthalpy). Besides, the model employed in the statistical part is lacking two factors that a priori should improve the modelization. The first one is the unpolarizable character of the hydrate, which neglects the actual response of the first-shell water molecules to the perturbation produced mainly by the second-shell waters. Nevertheless, this shortcoming should not be too important because this effect has been partially taken into account, in an averaged way, by polarizing the hydrate with a solvent reaction field when the HIW potential was developed. A second limitation of our modelization is the consideration of rigid body for both the hydrate and water molecules. This factor limits the type of properties which can be investigated by the implicit rigid approach. Intramolecular dynamic properties would need further developments. In any case, the main geometrical distortions affecting the intermolecular interactions have been considered in the present modelization. Thus, the distance between the cation and the first-shell water molecules, as well as the whole molecular geometry of these waters, has been quantum mechanically computed. Marx et al., using the $a b$ initio molecular dynamics method, have observed that the geometrical structure for water molecules beyond the first hydration shell of $\mathrm{Be}^{2+}$ is very similar to that of pure bulk water obtained with the same methodology. ${ }^{77} \mathrm{~A}$ different source of uncertainty in the close agreement comes from the experimental value of $\Delta H_{\text {hydr }}\left(\mathrm{Cr}^{3+}\right)$. It is not a directly measured quantity, but rather it is a quantity based on an extra thermodynamics assumption. ${ }^{78}$ As a general rule, it is difficult to imagine hidden error cancellations among the different employed approaches larger than $2 \%-4 \%$ of the final value. Therefore, it seems reasonable to conclude that the combination of the potentials presented in this work are able to supply a fairly good description of the energetics of the $\mathrm{Cr}^{3+}$ ionic solution.

The previous evaluation ${ }^{39}$ of $\Delta H_{\text {hydr }}\left(\mathrm{Cr}^{3+}\right)$ using the HIW + MCY potentials and the same computational conditions was $-1259.1 \mathrm{kcal} / \mathrm{mol}$, that is an overestimation of $\sim 140 \mathrm{kcal} / \mathrm{mol}$. The main difference between the previous value and that obtained here comes from the statistical estimation of the hydration for the hydrated ion, $\Delta H_{\text {hydr }}\left(\left[\mathrm{Cr}\left(\mathrm{H}_{2} \mathrm{O}\right)_{6}\right]^{3+}\right)$, which goes from $\sim-710 \mathrm{kcal} / \mathrm{mol}$ with the HIW+MCY potentials to $-573 \mathrm{kcal} / \mathrm{mol}$ with the $\mathrm{HIW}_{P}+\mathrm{MCHO}$ ones. The improvement reached may be interpreted as a consequence of the importance that nonadditive and polarization terms beyond the first hydration shell do have for highly charged ions. A first indication of the improvement can be obtained from the better estimation of the many-body terms directly joined to the second hydration shell in the $\mathrm{Cr}^{3+}$ isolated cluster. However, this value is estimated to be $\sim 40 \mathrm{kcal} / \mathrm{mol}$ whereas the results from the simulation of the solution leads the value to $\sim 140 \mathrm{kcal} / \mathrm{mol}$. This indicates that polarization effects, although less intense than for the first and second shell phenomena, affect a large number of solvent molecules of the system. Likewise, slight geometrical changes in the average distances of the successive solvation shells as a consequence of the increasing repulsion among the polarized water molecules of a given hydration shell, lead to the modification of the electrostatic contribution to the total interaction energy. Their consideration in this work has been a key point to carefully improve the description of the energetics of the $\mathrm{Cr}^{3+}$ hydration.

In conclusion, the ability of the presented model to give satisfactory structural and energetic answers on highly charged ionic solutions supports the idea that first-principles interaction potentials which are improved in a systematic stepwise procedure hold up. In fact, the adaptation of the hydrated ion-water interaction potential to be used in a polarizable environment has been easily done and no spurious effects have appeared. This seems to indicate the soundness of the hydrated ion approach to develop first-principles interaction potentials. Likewise, the MCHO water model has been revealed as a well-balanced model of water for describing environments as strong as those of the second hydration shell of a trivalent monoatomic cation. Future developments should be addressed in a double sense, on one hand toward the prospect of methods to generalize the inclusion of polarization effects to others cations without needing the refitting of the potential surface, and on the other hand, toward increasing the properties accessible to this method by the inclusion of flexible models for water and hydrate, and their coupling to molecular dynamics simulations.

\section{ACKNOWLEDGMENTS}

We thank Spanish DGICYT (PB95-0549) as well as DGAPA-UNAM (ES-112896) and CONACyT (L004-E) for financial support. J.M.M. thanks I.O.B. and H.S.M. for their hospitality during his stay at Cuernavaca. J.H.C. is thankful to E.S.M. and J.M.M. for their warm hospitality during his stay at Sevilla. Dr. Keith Refson, Oxford University, is thanked for useful comments. 
${ }^{1}$ J. Burgess, Metal Ions in Solution (Ellis Horwood, New York, 1978).

${ }^{2}$ B. E. Conway, Ionic Hydration in Chemistry and Biophysics. Studies in Physical and Theoretical Chemistry (Elsevier, Amsterdam, 1981), Vol. 12.

${ }^{3}$ J. M. G. Barthel, H. Krienke, and W. Kunz, Physical Chemistry of Electrolyte Solutions (Steinkopff, Darmstadt, 1998).

${ }^{4}$ A. Warshel, Computer Modeling of Chemical Reactions in Enzymes and Solutions (Wiley, New York, 1991).

${ }^{5}$ D. T. Richens, The Chemistry of Aqua Ions (Wiley, Chichester, 1997).

${ }^{6}$ M. P. Allen and D. J. Tildesley, Computer Simulation of Liquids (Oxford University Press, Oxford, 1987).

${ }^{7}$ D. Frenkel and B. Smit, Understanding Molecular Simulations: From Algorithms to Applications (Academic, San Diego, 1996).

${ }^{8}$ A. J. Stone, The Theory of Intermolecular Forces (Clarendon, Oxford, 1996).

${ }^{9}$ E. Clementi, in Modern Techniques in Computational Chemistry (Escom, Leiden, 1990), Chap. I.

${ }^{10}$ N. J. Elrod and R. J. Saykally, Chem. Rev. 94, 1975 (1994).

${ }^{11}$ E. Clementi, G. Corongiu, B. Jönsson, and S. Ramano, J. Chem. Phys. 72, 260 (1980)

${ }^{12}$ M. Migliore, G. Corongiu, E. Clementi, and G. Lie, J. Chem. Phys. 88, 7766 (1988).

${ }^{13}$ L. A. Curtiss, J. W. Halley, J. Hautman, and A. Rahman, J. Chem. Phys. 86, 2319 (1987).

${ }^{14}$ I. Ortega-Blake, O. Novaro, A. Lés, and S. Rybak, J. Chem. Phys. 76, 5405 (1982).

${ }^{15}$ I. Ortega-Blake, J. Hernández-Cobos, and O. Novaro, J. Chem. Phys. 81, 1894 (1984).

${ }^{16}$ P. Maye and M. Mezei, J. Mol. Struct.: THEOCHEM 362, 317 (1996).

${ }^{17}$ P. Pérez, W. K. Lee, and E. W. Prohofsky, J. Am. Chem. Soc. 113, 481 (1991).

${ }^{18}$ L. Perera, U. Essmann, and M. L. Berkowitz, J. Chem. Phys. 102, 450 (1995).

${ }^{19}$ L. X. Dang, J. E. Rice, J. Caldwell, and P. A. Kollman, J. Am. Chem. Soc. 113, 2481 (1991).

${ }^{20}$ G. Corongiu, M. Migliore, and E. Clementi, J. Chem. Phys. 90, 4629 (1989).

${ }^{21}$ M. Sprik, M. L. Klein, and K. Watanabe, J. Phys. Chem. 94, 6483 (1990).

${ }^{22}$ J. Caldwell, L. X. Dang, and P. A. Kollman, J. Am. Chem. Soc. 12, 9144 (1990).

${ }^{23}$ L. X. Dang and D. E. Smith, J. Chem. Phys. 99, 6950 (1993).

${ }^{24}$ R. A. Bryce, M. A. Vincent, N. O. J. Malcolm, I. H. Hillier, and N. A. Burton, J. Chem. Phys. 109, 3077 (1998).

${ }^{25}$ M. I. Bernal-Uruchurtu and I. Ortega-Blake, J. Chem. Phys. 103, 1588 (1995).

${ }^{26}$ M. I. Bernal-Uruchurtu, J. Hernández-Cobos, and I. Ortega-Blake, J. Chem. Phys. 108, 1750 (1998).

${ }^{27}$ M. M. Probst, E. Spohr, and K. Heinzinger, Chem. Phys. Lett. 161, 405 (1989).

${ }^{28}$ G. W. Marini, N. R. Texler, and B. M. Rode, J. Phys. Chem. 100, 6808 (1996).

${ }^{29}$ Y. P. Yongyai, S. Kokpol, and B. M. Rode, J. Chem. Soc., Faraday Trans. 88, 1537 (1992).

${ }^{30}$ L. A. Curtiss and R. Jurgens, J. Phys. Chem. 94, 5509 (1990).

${ }^{31}$ M. N. D. S. Cordeiro, J. A. N. F. Gomes, A. González-Lafont, J. M. Lluch, and J. Bertrán, Chem. Phys. 141, 379 (1990).

${ }^{32}$ T. Kowall, F. Foglia, L. Helm, and A. E. Merbach, J. Am. Chem. Soc. 117, 3790 (1995).

${ }^{33}$ G. Corongiu and E. Clementi, J. Chem. Phys. 69, 4885 (1978).

${ }^{34}$ E. Sánchez Marcos, R. Pappalardo, J. Barthelat, and F. Gadea, J. Phys. Chem. 96, 516 (1992).

${ }^{35}$ M. Cossi and M. Persico, Theor. Chim. Acta 81, 157 (1991).

${ }^{36}$ I. Ortega-Blake, A. Lés, and G. del Conde, J. Chem. Phys. 73, 5698 (1980).

${ }^{37}$ R. R. Pappalardo and E. Sánchez Marcos, J. Phys. Chem. 97, 4500 (1993).

${ }^{38}$ E. Sánchez Marcos, J. Martínez, and R. Pappalardo, J. Chem. Phys. 105, 5968 (1996).
${ }^{39}$ R. R. Pappalardo, J. M. Martínez, and E. Sánchez Marcos, J. Phys. Chem. 100, 11748 (1996).

${ }^{40}$ J. M. Martínez, R. R. Pappalardo, E. Sánchez Marcos, K. Refson, S. Díaz-Moreno, and A. Muñoz-Páez, J. Phys. Chem. B 102, 3272 (1998).

${ }^{41}$ J. M. Martínez, R. R. Pappalardo, and E. Sánchez Marcos, J. Chem. Phys. 109, 1445 (1998).

${ }^{42}$ J. M. Martínez, R. R. Pappalardo, and E. Sánchez Marcos, J. Am. Chem. Soc. 121, 3175 (1999).

${ }^{43}$ A. Bleuzen, F. Foglia, E. Huret, L. Helm, A. E. Merbach, and J. Weber, J. Am. Chem. Soc. 118, 12777 (1996).

${ }^{44}$ E. Wasserman, J. R. Rustad, and S. Xantheas, J. Chem. Phys. 106, 9769 (1997).

${ }^{45}$ X. Periole, D. Allouche, J. P. Daudey, and Y. H. Sanejouand, J. Phys. Chem. B 101, 5018 (1997).

${ }^{46}$ X. Periole, D. Allouche, A. Ramírez-Solís, I. Ortega-Blake, J. P. Daudey, and Y. H. Sanejouand, J. Phys. Chem. B 102, 8579 (1998).

${ }^{47}$ M. N. D. S. Cordeiro and J. A. N. F. Gomes, J. Comput. Chem. 14, 629 (1993).

${ }^{48}$ O. Matsuoka, E. Clementi, and M. Yoshimine, J. Chem. Phys. 64, 1351 (1976).

${ }^{49}$ W. L. Jorgensen, J. Chandrasekhar, J. D. Madura, R. W. Impey, and M. L. Klein, J. Chem. Phys. 79, 926 (1983).

${ }^{50}$ J. R. Rustad, B. P. Hay, and J. W. Halley, J. Chem. Phys. 102, 427 (1995).

${ }^{51}$ J. W. Halley, J. R. Rustad, and A. Rahman, J. Chem. Phys. 98, 4110 (1993).

${ }^{52}$ H. Saint-Martin, C. Medina-Llanos, and I. Ortega-Blake, J. Chem. Phys. 93, 6448 (1990).

${ }^{53}$ J. Hernández-Cobos and I. Ortega-Blake, J. Chem. Phys. 99, 9122 (1993).

${ }^{54}$ J. Hernández-Cobos and I. Ortega-Blake, J. Chem. Phys. 103, 1 (1995).

${ }^{55}$ H. Taube, J. Phys. Chem. 58, 523 (1954).

${ }^{56}$ H. Ohtaki and T. Radnai, Chem. Rev. 93, 1157 (1993).

${ }^{57}$ C. Breneman and K. Wiberg, J. Comput. Chem. 11, 361 (1990).

${ }^{58}$ M. Sprik and M. L. Klein, J. Chem. Phys. 89, 7556 (1988).

${ }^{59}$ L. X. Dang, J. Chem. Phys. 97, 2659 (1992).

${ }^{60}$ S. W. Rick and B. J. Berne, J. Am. Chem. Soc. 118, 672 (1996).

${ }^{61}$ J. M. Martínez, R. R. Pappalardo, and E. Sánchez Marcos, J. Chem. Phys. 110, 1669 (1999)

${ }^{62}$ M. M. Probst, Chem. Phys. Lett. 137, 229 (1987).

${ }^{63}$ N. Metropolis, A. W. Rosenbluth, M. N. Rosenbluth, A. H. Teller, and E. Teller, J. Chem. Phys. 21, 1087 (1953).

${ }^{64}$ J. Hernández-Cobos, H. Saint-Martin, and I. Ortega-Blake, MONTECUERNA, 1993. This library is available upon request at MONTCUER@ce.ifisicam.unam.mx.

${ }^{65} \mathrm{~K}$. Refson, MOLDY User's Manual Rev. 2.11. MOLDY code can be obtained from the CCP5 program library, or by anonymous ftp from ftp.earth.ox.ac.uk.

${ }^{66}$ J. E. Roberts and J. Schnitker, J. Phys. Chem. 99, 1322 (1995).

${ }^{67}$ J. M. Martínez, R. R. Pappalardo, and E. Sánchez Marcos, J. Phys. Chem. A 101, 4444 (1997).

${ }^{68}$ M. Pavlov, P. E. M. Siegbahn, and M. Sandström, J. Phys. Chem. A 102, 219 (1998).

${ }^{69}$ H. S. Frank and M. W. Evans, J. Chem. Phys. 13, 507 (1945).

${ }^{70}$ H. Saint-Martin and I. Ortega-Blake, in Biomolecules in Organic Solvents, edited by A. Gómez-Puyou (CRC, Boca Raton, FL, 1992), Chap. 3.

${ }^{71}$ M. C. Read and M. Sandström, Acta Chem. Scand. 46, 1177 (1992).

${ }^{72}$ R. Caminiti, G. Licheri, G. Piccaluga, and G. Pinna, J. Chem. Phys. 69, 1 (1978).

${ }^{73}$ R. D. Broadbent, G. W. Neilson, and M. Sandström, J. Phys.: Condens. Matter 4, 639 (1992).

${ }^{74}$ A. Muñoz-Páez, R. R. Pappalardo, and E. Sánchez Marcos, J. Am. Chem. Soc. 117, 11710 (1995).

${ }^{75}$ P. Bergstrom, J. Lindgren, M. Read, and M. Sandström, J. Phys. Chem. 95, 7650 (1991).

${ }^{76}$ Y. Marcus, J. Chem. Soc., Faraday Trans. 83, 339 (1987), and references therein.

${ }^{77}$ D. Marx, M. Sprik, and M. Parrinello, Chem. Phys. Lett. 273, 360 (1997).

${ }^{78}$ Y. Marcus, Ion Solvation (Wiley, Chichester, 1986). 